\title{
MedienPädagogik
}

Zeitschrift für Theorie und Praxis der Medienbildung

\section{Learning Analytics im Kontext komplexer Fallsimulationen personenbezogener Dienstleistungsberufe}

\author{
Chancen und Begrenzungen der Lernstandsanalyse im Serious Game
}

Cornelia Jeremias-Pölking, Cornelius Knab, Bernward Hoffmann, Nadin Dütthorn, Manfred Hülsken-Giesler, Miriam Peters und Rasmus Pechuel

\section{Zusammenfassung}

Der Beitrag diskutiert anhand des Forschungsprojektes Game Based Learning in Nursing - Spielerisch Lernen in authentischen, digitalen Pflegesimulationen (GaBaLEARN) die Einbindung von Learning Analytics in digitale Fallsimulationen eines Serious Games. Es werden pflege- und mediendidaktische Impulse zur Entwicklung und Integration von Learning Analytics für die Darstellung von situativen, komplexen Kompetenzen im Bereich personenbezogener Dienstleistungsberufe, wie der Pflege, dargestellt.

Learning Analytics in the context of authentic, complex and multidimensional scenarios of nursing education. Chances and limitations of learning analytics in a serious game

\begin{abstract}
The article discusses the integration of learning analytics in digital simulations of authentic scenarios of a serious game. The principles of nursing education lead to show how authentic, complex and multidimensional narratives are used to facilitate learning processes in humanties. This takes place using the example of the research project Game Based Learning in Nursing - Spielerisch Lernen in authentischen, digitalen Pflegesimulationen.

1. Einleitung: Lernen - Analyse - digitale Daten

Lernen gehört zu den Zentralbegriffen der Erziehungswissenschaft und ist vielleicht das Kernelement pädagogischen Handelns und dessen Reflexion. In intentionalen Lehr-Lernprozessen kümmert sich die Didaktik um die Auswahl der Inhalte und die Methodik will deren Vermittlung ermöglichen oder erleichtern. Im Kontext beruflichen Lernens und das konkret im Bereich personenbezogener Dienstleistungen kann Lernen als Zugewinn beruflicher Handlungskompetenz verstanden werden.
\end{abstract}


Die Kompetenzorientierung (Erpenbeck, Grote, und Sauter 2017, XII) dominiert ohnehin seit mehr als einem Jahrzehnt das Nachdenken über intentionale Lernprozesse in Kontexten formaler Bildung. Konstruktivistische Didaktik macht dabei deutlich: Lernen ist eine Aktivität des Lernenden auf der Basis eines Angebotes oder Reizes, aber keine Vermittlungsleistung des Lehrenden oder gar des Lehrmaterials. Der Gegenstand des Lernens bzw. das Lernangebot stellen nur den Reiz dar, den der Lernende sich selbst aneignen, den er selbst für sich konstruieren muss (Schulmeister 2007, 68). Das gilt auch für jede Form digitalisierter Lernanregungen (Kerres 2018, 163) und somit auch für das im späteren Verlauf beschriebene Forschungsprojekt mit seinem Serious Game «Take Care».

Gerade das Verständnis konstruktivistischen Lernens ermöglicht die Perspektive von Emotionalität, Leiblichkeit, Interaktion und Situiertheit des Handelns und die Perspektive von Selbststeuerung im Lernprozess. Didaktische Designs in diesem Zusammenhang fokussieren insbesondere auf die Einbindung des Anwendungskontextes und der Authentizität (Kerres 2018, 162), was als wesentlich für das hier beschriebene Projekt verstanden wird. Das Lernen im Rahmen der digitalen Anwendung bezieht sich hier auf Prozesse des Entdeckens und des Erfahrens. Im Rahmen des Entdeckens werden Erfahrungen gesammelt und aktiv zur Konstruktion von Wissen eingesetzt. Dabei bedingt die gemachte Erfahrung das Lernen bzw. das Gelernte die zu machende Erfahrung in all ihrer Prozesshaftigkeit (Buck 2019, 5f.) auch in digitalen Lern- und Erfahrungswelten.

Lernen hat, das machen sowohl die Kompetenzdebatte wie auch teils alte Überlegungen zu Handlungsorientierung, Begreifen und Erfahrung deutlich, nicht nur, wahrscheinlich nicht einmal zuerst eine Wissensdimension, sondern es bedarf ebenfalls des Könnens und der Motivation zur Eigenaktivität. Im Kompetenzbegriff treffen die Aspekte Wissen, Können und Wollen notwendig zusammen.

Trotzdem bleibt die spannende Kernfrage: Was passiert im Individuum beim Prozess des Lernens? Ein Prozess, der nach Buck $(2019,3)$ zu den Verborgensten und Unbekanntesten gehört. Was passiert beim Kompetenzerwerb? Und an welchen Schrauben kann man drehen, um Lernen anzuregen, zu fördern, zu erleichtern, also den Lernerfolg zu verbessern - bestenfalls in Gestalt eines Kompetenzerwerbs? Da geht es um die alte didaktische Frage der Inhaltsauswahl (nicht zu schwer, nicht zu leicht, elementar, aber exemplarisch für grössere Zusammenhänge), um möglichst gutes Lernmaterial (anschaulich, konkret, aber auf Verallgemeinerung und Abstraktion hin offen), um Weckung von Motivation, Erzeugung und Erhalt von Aufmerksamkeit, um lernförderliche Umgebungen u.v.m.

Durch die gesellschaftlich rasant voranschreitenden Prozesse der Digitalisierung von immer mehr Dingen und Abläufen unseres Alltagslebens entstehen Mengen von Daten über menschliches Handeln, die vielfach gespeichert werden und ausgewertet werden können; letzteres bezeichnet der Begriff «Data Mining». Das nutzen die 
globalen Datenkraken wie Google, Facebook, Amazon, Apple etc. aus, um mit den Daten der Menschen, ihrer Verknüpfung und Analyse viel Geld zu erwirtschaften. Das ideelle Gut einer «informationellen Selbstbestimmung» des Menschen ist vielfach schon zur Farce verkommen, weil wir selbst mehr oder weniger bewusst und freiwillig an der Datenproduktion mitwirken: die bringt uns nämlich viele Bequemlichkeiten und Vorteile und die Risiken erscheinen (uns) nicht so schlimm. Dabei kann und darf es nicht darum gehen, Prozesse der Digitalisierung deswegen grundsätzlich zu verdammen. Sie bieten in der Tat sehr viele positive Chancen und wir kommen ohne sie in Gegenwart und Zukunft nicht zurecht - und können mit ihrer Hilfe sicher auch Lernen im Interesse des Individuums verbessern.

Digitalisierung hat längst auch das Feld des institutionellen Lernens erreicht, der konsequente Ausbau ist politisch gewollt und gefördert. Auch in traditionellen Lernformen etwa in der Schule gab es schon immer Daten über Lernverhalten, die gesammelt und ausgewertet wurden. Hausarbeitshefte, Klassenarbeiten, Klassenbücher, Zeugnisse etc. sind solche Datensammlungen und wertende Interpretationen. Die subjektive Komponente dieser Daten war niemals zu leugnen. Und die Sammlung und Verknüpfung dieser Daten war niemals so umfassend, dass daraus ein aussagekräftiger Datenschatten des Ichs zusammensetzbar war. Und trotzdem: Schulnoten und Schulversagen begleiten manche Menschen ein Leben lang.

Bei Lernprozessen, die digitalisierte Materialien, Lernimpulse, Aufgaben etc. verwenden und in digitalen Umgebungen ablaufen - also beispielsweise mit einem interaktiven Tool oder digitalen Schulbuch auf einem Tablet, innerhalb einer digitalen Serious-Game-Umgebung oder auf einer Lehr-Lern-Plattform wie Moodle, Ilias u. a. - entstehen viel mehr Daten, die scheinbar objektiv anfallen, ggf. erfasst werden und ausgewertet, also analysiert und interpretiert werden könnten; sie haben an der Oberfläche meist gar nichts mit Lernen zu tun.

Aber ihre Auswertung unter genau diesem Aspekt des Lernens wird seit einigen Jahren unter dem Begriff «Learning Analytics» verhandelt, allerdings bislang ohne einheitliche Begriffsbestimmung (Leitner und Ebner 2017, 371). Befürworter versprechen sich davon datengestützte, wissenschaftliche Lernstandsanalysen, auf deren quasi objektiver Basis man Lernprozesse besser verstehen und anschliessend optimaler gestalten könne (Buckingham Shum und Ferguson 2012, 3f.; Ifenthaler 2017, 367f.). 2011 wurde auf der ersten Conference on Learning Analytics and Knowledge eine seitdem häufig zitierte Definition formuliert:

"Learning analytics is the measurement, collection, analysis and reporting of data about learners and their contexts, for purposes of understanding and optimising learning and the environments in which it occurs.» (Ferguson 2012, 305). 
Das allgemeine Data Mining ist weit entwickelt und wird von vielen Akteuren eingesetzt und perfektioniert. Zur speziellen auf den Bildungsbereich fokussierten Form der Learning Analytics gibt es vor allem im Bereich beruflicher Bildung bisher nur wenige Diskussionen. Deshalb müssten praktische Erfahrungen und theoretische Diskussionen über die Grundlagen von Learning Analytics ineinandergreifen, um Art und Umfang von Datenerhebungen und -auswertungen in Erziehungswissenschaft und pädagogischer Praxis zu begründen und verantwortlich zu gestalten.

Im Rahmen des Forschungsprojektes Game Based Learning in Nursing - Spielerisch Lernen in authentischen, digitalen Pflegesimulationen (GaBaLEARN), gefördert vom Bundesministerium für Bildung und Forschung sowie dem europäischen Sozialfonds für Deutschland (http://eduproject.eu/gabalearn), geht es um die Entwicklung eines exemplarischen Serious Game und die Evaluation seiner Möglichkeiten. Dabei steht die Frage von Learning Analytics in der digital gestützten Pflegebildung quasi nebenbei an, wird aber in einem künftigen Projekt Grundsatzfragen und Gelingensbedingungen der digital-gestützten Lernstandsanalyse in Bildungskontexten der personenbezogenen Dienstleistung (LernStandPD) explizit aufgegriffen.

Im Projekt GaBaLEARN wurde ein Serious Game mit dem Titel «Take Care» konzeptioniert und in einer ersten spielbaren Version umgesetzt und evaluiert. Das Spiel ist für den Bereich der Pflegebildung gedacht. Der Terminus «Serious Game» wird häufig unspezifisch verwendet (Hoblitz 2015, 13ff.). Wir orientieren uns an einem weiten Verständnis des Begriffes unter Einbeziehung von Lernspielen (Educational Games) als Unterkategorie. «Serious Games» bezeichnen dann eine Kategorie von unterhaltsamen und interaktiven Bildungsprogrammen (Ganguin 2016, 3). Das Spielangebot geht dabei über den reinen Spielspass hinaus und dient «ernsthaften» Hintergründen oder Zwecken wie der Vermittlung von Inhalten und Wissen oder der Sensibilisierung für bestimmte Themen (Fromme, Biermann, und Unger 2010, 39). Durch das planvolle Einbinden bewährter Elemente digitaler Spiele soll Motivation geweckt und in nachhaltige Lernprozesse überführt werden (Unger, Goossens, und Becker 2015, 159). Die planvolle Einbindung der Lernintention kommt in der folgenden Definition zum Ausdruck: «Lernspiele sind Computerspiele, die explizit zur Iniitierung [sic!] bestimmter Lern- und Entwicklungsprozesse eingesetzt werden» (Wechselberger 2012a, 88).

Im Lernspiel «Take Care» geht es um die Lernbereiche Multimorbidität und Chronizität im Kontext eines Pflegewohnbereichs für an Demenz erkranke Menschen. Zielgruppe des Spiels sind Auszubildende in Berufen personenbezogener Dienstleistungen, insbesondere der Pflegeausbildung. Die Lernenden steuern die virtuelle Spielfigur einer Pflegekraft; sie bewegen sich im spieltypischen Zeitraffer über mehrere Tage und Schichten in interaktiven realitätsnahen Szenarien der Pflegestation. Dabei geht es kaum um pflegerische Tätigkeiten wie Waschen, Medikamente verteilen etc., sondern vor allem um Kommunikationssituationen mit Patienten, Angehörigen und 
Kolleginnen und Kollegen; dabei muss eine Pflegekraft ständig handeln und Entscheidungen treffen. Die Hypothese der Spielkonzeption ist etwa folgende: im interaktiven Spiel kann besser als in Fallbeschreibungen oder Videoaufnahmen eine alltagsnahe Situation abgebildet werden; zudem motiviert das Spiel die Lernenden zur eigenen Aktion, die zwar eine Handlung im Spielszenario ist, aber deren Alltagsnähe im Blended-Learning reflektiert werden kann. Dabei erwerben die Lernenden Handlungskompetenzen, indem sie sich mit den (möglichen) Motiven und Folgen ihres Handelns reflexiv auseinandersetzen; und dies geschieht auf der Basis authentischer Simulationen, aber vom realen Handlungsdruck selbst eines Praktikums oder einer Berufseinführung entlastet.

Der jeweilige Spielverlauf und vor allem das Verhalten der Spielenden und ihre Entscheidungen für bestimmte Handlungs- und Interaktionspfade mit den virtuellen Charakteren des Spiels können als Ausgangspunkt von Learning Analytics im Spiel «Take Care» genutzt werden. Beim derzeitigen Stand der Spielentwicklung können prinzipiell eine Reihe von Daten erhoben, gespeichert und als Lernanalysen ausgewertet werden. Die Konzeption sieht vor, Ergebnisse entsprechender Analyse zum Lernverhalten nicht nur dem einzelnen Lernenden als Feedback im Rahmen des Lernspiels zu präsentieren, sondern möglicherweise weiterführende Erkenntnisse mit Zustimmung der Lernenden auch in das face-to-face Unterrichtsgeschehen einzubinden. Im Format des Blended Learning werden die zuvor gesammelten und strukturierten Daten (Learning Analytics) aus der Spielhandlung für eine Reflexion der getroffenen Spielentscheidungen in der dazugehörigen Präsenzphase der theoretischen Pflegeausbildung genutzt. Spielverlauf und Konsequenzen der getroffenen Entscheidungen sollten von Lehrenden und Lernenden gemeinsam interpretiert werden mit der Intention, dass Handlungsalternativen das Kompetenzspektrum des jeweils Lernenden erweitern helfen. Diese praktische Konkretisierung von Learning Analytics am Beispiel eines konkreten Lernspiels kann exemplarisch für Lernkontexte der personenbezogenen Dienstleistungsberufe insgesamt gelesen werden. Sie bedarf allerdings parallel einer theoretischen Begründung und Reflexion.

Im Folgenden wird deshalb zunächst die datenwissenschaftliche Herkunft der «Learning Analytics» thematisiert, um das Konzept danach im Kontext der beruflichen Bildung in der Pflege bzw. allgemeiner der personenbezogenen Dienstleistungen zu diskutieren; besonders wichtig dabei ist eine kritische Reflexion auf die Erhebung und Nutzung der Daten, besonders der personenbezogenen. Abschliessend werden einige Perspektiven zu Nutzung von «Learning Analytics» mit dem Lernspiel «Take Care» benannt und ein Ausblick auf ein weiteres Projekt geöffnet. 


\section{Datenwissenschaftliche Konzeption von Learning Analytics}

Das Sammeln von Nutzerdaten als Voraussetzung von Learning Analytics (Becker 2013, 64; Ferguson 2012, 305), wird im Bereich des «Data Mining» längst praktiziert. Mit Hilfe von statistischen Algorithmen werden aus «Big Data» Trends, Muster oder Gesetzmässigkeiten abgeleitet; das kann zur Auswertung menschlichen Verhaltens (z. B. Kaufverhalten) genutzt werden, was wiederum zu ziemlich verlässlichen Prognosen führt. Menschliches Verhalten, so die unterschwellige Botschaft, ist berechenbar. Verhalten generell kann als «gelernt» angesehen werden, aber explizit geht es erst beim «Educational Data Mining» um Fragen des Lernens; eine begriffliche Abgrenzung zu Learning Analytics ist nicht immer möglich. Educational Data Mining strukturiert und ordnet technologiebasiert die im Bildungskontext gesammelten Daten, um sie für weitere Analysen und Prognosen fruchtbar zu machen. Ansätze der Learning Analytics akzentuieren stärker die pädagogische Intention, also z. B. die Vorhersage von Lernerfolgen und deren Unterstützung und die Rolle von Pädagogen im Bereich der Dateninterpretation (Buckingham Shum und Ferguson 2012., 4f.; Liu u. a. 2016, 34; Schön und Ebner 2013, o.S.). Die Rückkopplung der gewonnenen Erkenntnisse an die Lehrenden zum Verstehen des Lernprozesses und an die Lernenden zur Reflexion des Lernprozesses sind dabei wesentlich (Leitner und Ebner 2017, 381; Staudt 2017, 573f.). Diese Rückbindung ist für eine Didaktisierung von Lernangeboten zum Zweck des grösstmöglichen Lernerfolges (Kerres 2018, 119) besonders relevant.

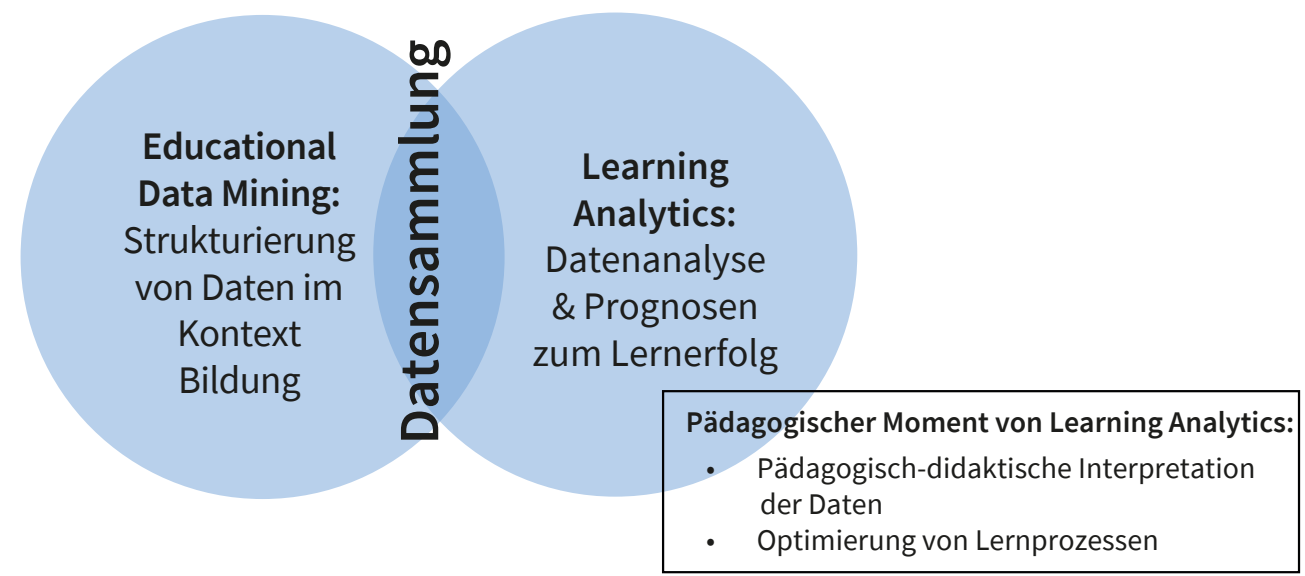

Abb. 1.: Educational Data Mining und Learning Analytics (Schön und Ebner 2013, o.S.; Leitner und Ebner 2017, 34; Buckingham Shum und Ferguson 2012, 4f.).

Zentrales Anliegen von Learning Analytics wäre somit, individuelle Lernprozesse besser zu verstehen, Lernleistungen vorherzusagen und Lernschwierigkeiten frühzeitig zu identifizieren. So könnten möglicherweise durch das Spiel «Take Care» Prozesse der Entscheidungsfindung sichtbar gemacht werden, um sie dem Lernenden bewusst und dem Lehrenden überhaupt erst zugänglich zu machen. Learning Analytics 
könnten neue Möglichkeiten für ein unmittelbares und individualisiertes Feedback an die Lernenden öffnen (Buckingham Shum und Ferguson 2012, 4f.; Ifenthaler 2017, 366; Kickmeier-Rust und Albert 2016, 35). Je nach unterrichtlichem Konzept und jeweiligem Kompetenzverständnis erhielte die Lehrperson so eine Möglichkeit, Lernende nach einem individuellen Kompetenzlevel einzuordnen und entsprechend ihres Kompetenzniveaus zu unterstützen (Taraghi u. a. 2017, 298f.).

Konkret können für Learning Analytics viele Daten genutzt werden, die bei der Nutzung digitaler Lernspiele anfallen: z. B. Zeitpunkt der Nutzung, Standortdaten, Navigationen in den Anwendungen, Mausbewegungen und Klicks (Becker 2013, 64f.; Liu u. a. 2016, 41). Das Ziel der Lernstandsanalysen impliziert die pädagogisch zu begründenden Entscheidungen darüber, welche Daten unter Berücksichtigung der Machbarkeit und der Datenethik gesammelt und analysiert werden sollen. Wenn es etwa darum geht, Informationen zum sozialen Miteinander einer Lerngruppe zu erhalten, könnten Daten zur Spielerinteraktion aus Gruppenchats relevant sein. Geht es um die Kompetenz zur Problemlösung, könnten Daten über die Verwendung von Tools und Werkzeugen in einem Videospiel gesammelt und ausgewertet werden (Liu u. a. 2016, 41). Voraussetzung jeder Art von Datensammlung und -strukturierung ist allerdings, dass der Gegenstand der Analyse klar vorstrukturiert ist, etwa durch ein Kompetenzmodell. Für die Analyse von weniger klar zu operationalisierendenden Lernzielen - etwa Empathie in sozialen Beziehungen oder Kompetenzen des Einzelfallverstehens - bedarf es noch weiterer Vorarbeiten, um angemessene Verfahren der Learning Analytics sinnvoll zur Anwendung zu bringen.

\section{Perspektiven auf Bedarfe und Nutzungsoptionen der Learning Analytics im Projekt GaBaLEARN}

Sind eine Operationalisierung und Messbarkeit von Kompetenzen bereits prinzipiell umstritten, ist die Nutzung von Learning Analytics dafür eine noch grössere Herausforderung. Im Kontext des Projektes GaBaLEARN verstehen wir Kompetenzen als personenbezogene Dispositionen zur strukturierten und sachbezogenen Selbstorganisation, die nur als Performanz in konkreten Situationen sichtbar werden (Erpenbeck, Grote, und Sauter 2017, XIIf.; Dütthorn 2014, 50ff.; Jung 2010, 20ff.). Im Spiel «Take Care» werden konkret alle mit Entscheidungen verbundenen Spielhandlungen auf der Grundlage eines speziell verwendeten Kompetenzmodells ${ }^{1}$ als Performanz gewertet, die auf Kompetenzen im Sinne von Dispositionen, also persönlichkeitsbezogenen Charakteristika, die zum Handlungsvollzug führen, verweisen. Diese lassen sich nur schwer operationalisieren. Ein Forschungsschwerpunkt im Rahmen des Folgeprojektes LernStandPD richtet sich daher unter anderem auf Aspekte der

1 Ein Artikel zum Kompetenzmodell aus GaBaLEARN befindet sich im finalen Schreibprozess zur Einreichung in PADUA (Fachzeitschrift für Pflegepädagogik, Patientenedukation und -bildung). 
Operationalisierung von Kompetenzen der Pflege, die letztlich in einer performativen Spielhandlung ausgewiesen werden sollen. Während bereits eine Vielzahl von Studien zum Lernen mit digitalen Medien im Allgemeinen vorliegt, wurden mediendidaktische Entwicklungen im Bereich der Serious Games mit integrierten Learning Analytics aufgrund der Diversität der Ansätze und dem Innovationsgrad bisher weitaus seltener evaluiert (Wang, Cuihua, und Ritterfeld 2009, 41ff.; Breuer 2010, 27f.; Schrader 2010, 179f.; Dörner u. a. 2016, V). Bisher vorliegende Untersuchungen beziehen sich auf die Entwicklung und lerntheoretische Begründung von Serious Games im Bereich der allgemeinbildenden Schulen; berufspädagogische Bildungsinteressen sind dabei kaum berücksichtigt (Wechselberger 2012b, 15ff.). Die Entwicklung und Evaluation von digitalen Lehr- und Lernarrangements, die auf spielerische Weise durch virtuelle Simulation komplexer Berufswelten realitätsnahes Lernen ermöglichen, und zusätzlich die Einbindung von Learning Analytics darin sind im deutschsprachigen Bildungsraum bislang kaum zu finden (Beutner und Pechuel 2015, 1f; Becker 2013, 115). Für den Bereich der beruflichen Bildung in Deutschland, insbesondere die personenbezogenen Dienstleistungsberufe gibt es kaum Entwicklungen von Serious Games und nach Recherchen im Projekt GaBaLEARN keine Serious Games in der deutschen Pflegeausbildung. Deshalb werden im Folgenden einige pflegewissenschaftliche und -didaktische Anforderungen an ein Serious Game im Berufsfeld Pflege entfaltet.

\subsection{Bedarfe und Möglichkeiten von Learning Analytics für personenbezogene Dienstleistungsberufe am Beispiel der Pflege}

Um die Herausforderungen für Learning Analytics im Kontext der Pflegebildung zu verstehen, sind zunächst die Besonderheiten und Herausforderungen der professionellen Pflege als personenbezogene Dienstleitung sowie einige Charakteristika des Berufsfeldes zu skizzieren.

Folgt man der Definition des internationalen Weltbundes der Pflegenden (International Council of Nurses, ICN), umfasst berufliche Pflege

«die eigenverantwortliche Versorgung und Betreuung, allein oder in Kooperation mit anderen Berufsangehörigen, von Menschen aller Altersgruppen, von Familien oder Lebensgemeinschaften, sowie von Gruppen und sozialen Gemeinschaften, ob krank oder gesund, in allen Lebenssituationen (Settings). Pflege schließt die Förderung der Gesundheit, Verhütung von Krankheiten und die Versorgung und Betreuung kranker, behinderter und sterbender Menschen ein. Weitere Schlüsselaufgaben der Pflege sind Wahrnehmung der Interessen und Bedürfnisse (Advocacy), Förderung einer sicheren Umgebung, Forschung, Mitwirkung in der Gestaltung der Gesundheitspolitik sowie im Management 
des Gesundheitswesens und in der Bildung.» («Die Bedeutung professioneller Pflege» 2002, o.S.) $)^{2}$

Berufliche Pflege ist somit als systematisches Handeln angelegt, das seine Begründung aus wissenschaftlichen Erkenntnissen zum Beispiel der Gesundheits- und Pflegeforschung (externe Evidenz) sowie im Rückgriff auf die spezifischen individuellen Präferenzen und Ziele der Hilfeempfänger (interne Evidenz) erhält.

Ein weiteres Ausbildungsziel der Pflege besteht darin, Kompetenzen im Zusammenhang mit der Recherche und Bewertung pflegerelevanten Wissens anzubahnen sowie die Lernenden dazu zu befähigen, angemessene Reaktionen und Problemlösungen für den berufsbezogenen Einzelfall abzuleiten. Entsprechende Lernprozesse sind dabei immer an die Komplexität und die Multiperspektivität beruflicher Arbeitsprozesse und realer beruflicher Kontexte zu binden (Darmann-Finck 2010, 169ff.; Greb 2010, 145f; Dütthorn und Gemballa 2013, 1f.). Die Fähigkeit zu einem auf Interaktion beruhenden Aushandlungsprozess in beruflichen Situationen gilt als eine Kernkompetenz professionell Pflegender (Hülsken-Giesler und Koporal 2013, 5f.; Hülsken-Giesler u. a. 2010, 224; Dütthorn 2014, 252ff.). Das erfordert neben einer ausgeprägten Reflexionsfähigkeit und Fachkompetenz immer auch Kompetenzen der situativen Urteilsfähigkeit und der Erfassung individueller Bedürfnisse der zu Pflegenden. Hier können Learning Analytics zum Spiel «Take Care» einen Beitrag leisten, indem Spielentscheidungen getrekkt werden. Somit kann abgebildet werden, inwiefern diese Multiperspektivität im Rahmen von Entscheidungen ihre Berücksichtigung findet.

Zukünftige Berufsangehörige sind im Rahmen der Pflegebildung auf einen «reflektierten Umgang mit objektiven Widersprüchen einer professionellen Pflege» vorzubereiten (Hülsken-Giesler und Koporal 2013, 5). Am Beispiel von «Take Care» besteht durch die Analyse des Spielverhaltens die Möglichkeit, berufliche Dilemmata im Spiel zu erleben und Strategien im Umgang mit diesen Dilemmata in der konkreten Situation zu erproben. Learning Analytics leisten in diesem Fall einen Beitrag zur Reflexion der Spielhandlung und einer Übertragung auf authentische Praxis. Das kann Lernende handlungsentlastet auf die späteren beruflichen Pflegesituationen mit ähnlichem Komplexitätsgehalt vorbereiten.

2 https://www.dbfk.de/de/themen/Bedeutung-professioneller-Pflege.php; Original unter https://www.icn. ch/nursing-policy/nursing-definitions. 


\subsection{Didaktische Perspektive auf die Kompetenzentwicklung und Kompetenzmessung durch Learning Analytics}

Digital unterstütztes Lernen fokussiert in der beruflichen Aus-, Fort- und Weiterbildung bisher weitestgehend auf Informationsweitergabe, respektive auf wissensbasierte Vermittlung von Medien- und Fachkompetenzen. Neugier, intuitives Gespür und perspektivenreiche Problemlösefähigkeit als Merkmale eines entdeckenden Lernens können in der aktuellen textbasierten Fallarbeit im berufsbezogenen Unterricht der Pflegebildung mit konstruierten, wenig komplexen Übungsfällen kaum umgesetzt werden. Weniger verbreitet ist die authentische, multiperspektivische Fallarbeit (Hülsken-Giesler 2016, 19; Darmann-Finck 2010, 207ff.; Greb 2010, 146ff.; Hundenborn 2007, 52ff.), welche nun mittels pflegedidaktisch begründeter digitaler Methoden ihre Anwendung finden sollen.

Der Ansatz, digitale Medien und deren implizite Möglichkeiten von Learning Analytics für eine Reflexion des Lernprozesses an sich zu nutzen, ist im Kontext der Pflegebildung neu. Damit wird es möglich, so die Annahme, pflegedidaktische Konzepte der authentischen, multiperspektivischen Fallarbeit (Darmann-Finck 2010, 207ff.; Greb 2010, 146ff.; Dütthorn 2014, 107f.) erstmals in Formen eines technikgestützten, arbeitsprozessorientierten, realitätsnahen Lehrens und Lernens zu überführen. Das Verständnis von beruflichem Lernen richtet sich bislang zu wenig auf ein solch arbeitsprozessnahes und für die pflegerischen Berufe entscheidendes Verstehen von Einzelfällen. Deshalb könnte die Entwicklung von authentischen, digitalen Fallsimulationen in Form von Serious Games einen innovativen Beitrag zur pflegedidaktischen Kompetenzentwicklung im Sinne des hermeneutischen Fallverstehens leisten. Den Ausgangspunkt von digitalen Lernprozessen bilden hier komplexe authentische Fälle, die didaktisch aufbereitet den Lernenden eine Transferförderung ermöglichen und intuitives Gespür zur komplexen Problemlösefähigkeit wecken. Authentische Fälle zeichnen sich insbesondere durch die Abbildung komplexer und multidimensionaler Pflegephänomene aus und sind entgegen konstruierter Fälle aus der Pflegepraxis entnommen. Sie nehmen Falldeutungen nicht vorweg, sondern stellen sich deutungsoffen dar, sodass der Lernende auf Grundlage des authentischen Falls Pflegestrategien auswählt. Authentische Fälle kommen ohne allwissenden Erzähler aus. Der Fall wird aus der Perspektive des Protagonisten geschildert. Als zentrale Kompetenz soll die Arbeit mit authentischen Fällen nicht nur die Fachkompetenz, sondern auch die Anbahnung des hermeneutischen Fallverstehens zum Ziel haben (Darmann 2004, 464f.).

Die Grundannahme des Projektes GaBaLEARN kann man folgendermassen formulieren: Das Medium eines Serious Game ermöglicht die realitätsnahe Simulation der komplexen, multidimensionalen Berufswirklichkeit; es bietet zudem die Möglichkeit, Handlungen und ihre Folgen nachzuvollziehen und im Sinne der Learning 
Analytics zusammen mit den Lernenden auszuwerten; das könnte eine kompetenzorientierte Aneignung dieser komplexen Berufswirklichkeit unterstützen.

Inwiefern diese Fälle im Serious Game auch authentisch dargestellt werden können bzw. eine Realitätsnähe vermitteln, konnte in einer Evaluation der Spieletests erhoben werden. Lehrende und Lernende berichteten überwiegend von authentischen Charakteren und Situationen, welche Ihnen passend zum realen Arbeitsalltag im Bereich der Pflege erscheinen.

Es konnte nachgewiesen werden, dass durch technische Simulationen von realen Arbeitssituationen die Lernenden Handlungsroutinen erwerben (Kerres, Bormann, und Vervenne 2009, 6f.). Der Anspruch an die Spielsituationen in «Take Care» gestaltet sich so, dass, insofern das Spiellevel auf ein mittleres Anforderungsprofil der jeweils Lernenden angepasst ist, sie nicht primär als Lernsituation erlebt wird. Im Projekt GaBaLEARN wird angenommen, dass die Lernenden beim Durchlaufen der Spiellevel, insbesondere durch die Möglichkeit, verschiedene Lösungswege ohne Gefahr ausprobieren zu können, Handlungsroutinen erwerben, die sich von der simulierten Spielwelt und nach einer erfolgten Reflexion auf die reale Pflege übertragen lassen. Die Anforderung an Learning Analytics wird es sein, die gewählten Lösungswege und Entscheidungen aufzuzeichnen und dem Lernenden im Spiel, aber auch im Blended Learning-Reflexionen nach dem Spiel, aufzuzeigen.

\subsection{Datennutzung und Datenschutz im Kontext von Learning Analytics}

Die beschriebenen Potentiale von Learning Analytics erfordern generell, aber besonders im Kontext der personenbezogenen Dienstleistungen eine begleitend kritische Reflexion der Datennutzung und -auswertung sowie generell des Datenschutzes. Grundsätzlich dürfen Daten nur begründet und in angemessenem Umfang erhoben werden. Personenbezogene Daten der Nutzer müssen verlässlich geschützt sein. Pardo und Siemens (2014) halten folgende vier Elemente für zentral: «transparency, student control over the data, security, and accountability and assessment» (zitiert nach Chatti, u. a. 2014, 10).

Dabei geht es nicht nur um Rechtssicherheit etwa unter Beachtung des europäischen Datenschutzgrundverordnung (Europäisches Parlament und Rat der europäischen Union 2016, Art. 12-21), sondern auch um die dort formulierten Grundregeln für personenbezogene Daten wie Datensparsamkeit, Zweckbestimmung und Transparenz der Datenerhebung und nicht zuletzt die Frage von Persönlichkeitsrechten und Datensicherheit. Eine Erhebung von Daten nach dem Prinzip, was technisch machbar und möglicherweise für eine wissenschaftliche und pädagogische Analyse spannend wäre, greift erheblich zu kurz. Nicht nur der rechtliche Rahmen muss auf jeden Fall eingehalten werden, die Datenerhebung muss auch ethisch vertretbar sein. Und dass schliesst insbesondere eine pädagogische Ethik ein, die den Lernenden und seine Interessen in den Mittelpunkt stellt. 
Neben den Lehrenden und Lernenden haben möglicherweise weitere Instanzen im Bildungssystem, gewerbliche Unternehmen im Bildungssektor, aber auch politische Akteure ein Interesse an Daten, die für Learning Analytics gesammelt werden. Eine solche Nutzung muss unterbunden werden. Learning Analytics sollten somit im geschützten Lernraum zwischen Lernenden und Lehrenden verbleiben (Schulmeister 2013, 44). Learning Analytics als Technologie bzw. Techniktext stellen nach Swertz (2018) Machmittel dar. Macht ist hier sowohl positiv als auch negativ gemeint und in der pädagogischen Praxis nie gänzlich zu vermeiden (Swertz 2018, 2). Dieser Hinweis mahnt jedoch an Learning Analytics auch im geschützten Raum zwischen Lehrenden und Lernenden immer wieder zu hinterfragen und sich insbesondere als Lehrender und somit eher Machtausübender zu reflektieren, wie und inwiefern durch Learning Analytics machtgeladene Lehr-Lernsituationen entstehen und Nachteile für Lernende zu verhindern.

Im Lernkontext muss man nicht nur rechtlichen Ansprüchen genügen und ethische Aspekte berücksichtigen, sondern insbesondere das Vertrauen der Nutzer gewinnen und dessen Missbrauch kategorisch ausschliessen.

\section{4. Überlegungen zur Umsetzung von Learning Analytics in GaBaLEARN}

In einem Forschungsprojekt wie GaBaLEARN ergibt sich nun ein Problem: Neugier und offener Forschungsprozess geraten $u$. U. in Konflikt mit den Regeln der Datennutzung. Man weiss normalerweise beim Start und im Verlauf eines solchen Projektes noch nicht, welche Daten denn für Lernende oder Lehrende von Interesse sein könnten und Rückschlüsse auf den Lernprozess geben könnten. Das verführt dazu, erst einmal möglichst viele Daten zu erheben. Deshalb gilt ein Grundsatz auch für die Forschungs- und Evaluationsphase: die Datenerhebung muss für den Nutzer absolut transparent sein. Für eine spätere Veröffentlichung des Spiels und weitere wissenschaftliche bzw. pädagogische Arbeit damit müssen die Fragen der Datennutzung und die Begründung der Datenerhebung geklärt sein.

Leitend für die Auswahl der Daten ist für uns die Zielsetzung des Spiels: den Lernenden ihren Lernprozess transparenter zu machen und ihnen die Möglichkeit zu geben, ihre Lernstrategien und ihr Vorgehen zu verstehen, zu analysieren und zu optimieren. Die Daten sollen für die Lernenden insbesondere einen Anlass zur Selbstreflexion bieten (Swertz 2018, 4). Auf Seiten der Lehrenden sollten Daten dazu helfen, dass diese die individuellen Bedarfe von Lernenden oder auch von Gruppen besser erkennen und besser auf diese eingehen können. Das ist eine durchaus nicht konfliktfreie Perspektive. Denn auch bei noch so «wohlmeinender Haltung» des Lehrenden im Interesse seiner Schüler - die Lernenden müssen prinzipiell wissen, was die Lehrpersonen den Spielhandlungen an Informationen über den einzelnen Spieler entnehmen können. Manche Konkretionen, das wurde im Projekt GaBaLEARN bald 
klar, werden erst in der weiteren Projekterprobung konkret beantwortet werden können. Zentral sind neben dem Datenschutz zwei Fragerichtungen:

1. Welche Daten können während des Spielprozesses für welchen Zweck sinnvoll erhoben und nutzbar gemacht werden, ohne (a) den Spielfluss zu brechen und (b) das Verhalten des Spielers zu beeinflussen?

2. Welche Daten können aus dem Spielverlauf für eine reflexive Verwertung im Blended-Learning-Prozess sinnvoll erhoben und genutzt werden?

In den derzeitigen Überlegungen sind folgende Datenkategorien im Blick:

- Welche Dialogoptionen wählt der Lernende aus den verfügbaren Möglichkeiten aus? Gibt es dabei ein System? Ist ihm dies bewusst bzw. wie begründet er/sie in der anschliessenden Reflexion die Entscheidungen?

- Welche Aktionen führt der Lernende in welcher Reihenfolge im Spiel aus?

- Die im Spiel angefertigte Dokumentation des Lernenden sollte für eine anschliessende Reflexion verfügbar sein.

- Spielzeiten und -dauern könnten von Interesse sein, müssten aber ggf. nicht personalisiert erhoben werden.

Diese Datenkategorien können bei näherer Betrachtung in viele einzelne Daten unterteilt werden, und es wird eine Herausforderung sein, aussagekräftige Daten unter Beachtung der einleitend benannten Aspekte zu sammeln und auszuwerten. Auch muss die Auswertung der Daten nochmals in Bezug gesetzt werden zum globalen Lernziel: Zugewinn beruflicher Handlungskompetenz im Sinne einer hermeneutischen Einzelfallverstehens.

Bei der Verbindung von Datenanalysen mit Lernprozessanalysen schwingen natürlich Grundannahmen mit, die kritisch reflektiert werden müssen:

1. Das Spielverhalten der Lernenden wird als Lernverhalten gedeutet.

2. Um einen Kompetenzgewinn im Sinne von Learning Analytics nachweisen zu können, ist ein Kompetenzmodell zu hinterlegen, das Kompetenzen auf die SpielHandlungsebene hin operationalisiert. Erst auf dieser Basis kann das Spielverhalten und können die Lernwege des Spielers systematisch gemessen, analysiert und im Sinne des Kompetenzmodells ausgewertet werden.

3. Es sind Parameter im Spielhandeln zu bestimmen, anhand derer ein Kompetenzgewinn im Sinne des Kompetenzmodells interpretierend ermittelt werden kann.

4. Die genutzten Parameter sind logisch sinnvoll mit Algorithmen zu verknüpfen

5. Letztlich müssen die Lernenden entscheiden, ob diese Verknüpfungen für sie nachvollziehbar und weiterführend sind.

6. Beim Game geht es immer auch um Spielspass, der beim Serious-Game die Lernmotivation befördern soll. Learning Analytics sollten also auch Informationen bereitstellen, die Hinweise zur Motivation, zur Spielfreude und einem damit 
einhergehenden Lernerfolg ermöglichen. Motivation/Spielspass lässt sich anhand von einigen Daten indirekt messen, zum Beispiel an Spieldauer, an wiederholtem Starten des Spiels, an zielorientierten Strategien und an der im Spiel integrierten Kommunikation mit anderen Spielern.

Angelehnt an erste Evaluationen werden gezielte Belohnungssysteme im Spiel integriert, die das Spielerverhalten steuern sollen. Belohnungen im Spiel werten Daten des Spielverhaltens aus. Sie müssen dem Spieler in der Übertragung auf ihre Relevanz für eine dahinterliegende Alltagssituation bewusstgemacht werden. Der Spieler agiert als Pflegeperson und wird in dieser Rolle entsprechend bewertet. Das ist ein schwieriger Diskussions- und Entwicklungsprozess bei der Spielentwicklung.

Mit der Anlage und Programmierung des Spiels ist weitgehend algorithmisch zu hinterlegen, was ein «optimaler Ablauf» ist; dass heisst, die Bewertung des Handelns muss im Spiel soweit vorprogrammiert sein, dass sie analytisch dem Spieler als spielimmanente Belohnung oder als Feedback-System gespiegelt wird. Darüber hinaus werden die verschiedenen Abläufe mit einem Punktesystem versehen, sodass neben der Bewertung in richtig und falsch auch ein differenziertes Feedback möglich ist. Schliesslich soll und muss es auch eine zweite analytische Ebene geben: eine Art Blended-Learning-Kombination zwischen Spielerfahrung und -auswertung und Reflexionsgespräch in der Lernergruppe. Es gilt trotz aller Programmierbarkeit nicht die didaktische Grundlegung aus dem Blick zu verlieren, die vielfältige Falldeutungen vorsieht und die es auch zu belohnen bzw. zu bewerten gilt.

Wann im Verlauf des Spielgeschehens und in welcher Weise der Spieler Rückmeldung zur Auswertung und Bewertung seines Spielverhaltens bekommt, ist eine vieldimensionale Entscheidung. Auf jeden Fall sollte der Spielfluss nicht unterbrochen werden. Eine Auswertung beziehungsweise ein Aufzeigen der Entscheidungspfade ist erst am Ende des Spiels vorgesehen. Ein Kernproblem wird die Unterscheidung von Spielverhalten und antizipiertem Realverhalten sein. Es sollte hierbei vermieden werden, dass der Spieler sich vor allem spielstrategisch und nachrangig situationsadäquat verhält. Das kann zum Beispiel durch algorithmisch gesteuerte Zufallsaufgaben erfolgen, die der Spieler nicht kalkulieren kann; dies entspricht durchaus dem Alltag der beruflichen Arbeit.

\section{Ausblick}

Die Entwicklung eines Serious Game im Rahmen des Forschungsprojektes GaBaLEARN orientiert sich inhaltlich an realen Fallsimulationen. Das bedeutet, der Spieler muss sich wie im Berufsalltag immer wieder zwischen Handlungsalternativen entscheiden. Jede dieser Entscheidung hat Folgen, wobei es (entgegen algorithmischer Lösungen) inhaltlich/didaktisch keine deutliche Trennung in «richtig) und (falsch» 
gibt. Im Spiel ist es wichtig, authentische Situationen zu schaffen, da auch im Arbeitsalltag Entscheidungssituationen mit Konsequenzen auftreten. Im Gegensatz zum Spiel unterbleibt im Alltag allerdings häufig die Auswertung der gemachten Erfahrung oder ist auf gelegentliche Supervision verlagert. Deshalb muss das Spiel in einer sinnvollen Auswertung in Blended-Learning-Prozesse eingebunden sein. Learning Analytics sollen ein Auswertungsverfahren ermöglichen, das Lernenden ein besseres Verständnis der im Spiel erlebten Situationen erlaubt und sie durch eine Reflexionsphase führt und diese mit Daten untermauert. Lernende sollen sich Gedanken machen können, an welchen Stellen ihre Entscheidungen hätten anders sein können und zu welchem Zeitpunkt sie die Situation verstanden und entsprechend gehandelt haben und wann nicht.

Eine solche Orientierung von Learning Analytics bietet Möglichkeiten, Lernprozesse sichtbar zu machen, sie zu verstehen und gegebenenfalls auch zu optimieren. Eine Möglichkeit die ein ausschliessliches Lernfeedback über eine Lehrperson in solch objektiver Weise ohne die Grundlage der gesammelten Daten nicht bieten würde und im Sinne des Endeckenden Lernens und des Lernens aus Erfahrung viele Deutungen vorwegnehmen würde. Der Lernprozess soll nach Buck $(2019,5)$ im Zusammenhang von Lernen und Erfahrung mitbetrachtet werden. Learning Analytics vermögen hier im Rahmen der Auslese der gewählten Entscheidungen des Spielenden in «Take Care» gerade diesen Lern- und Erfahrungsprozess ein Stückweit offen zu legen. Dies ist nicht nur für erziehungswissenschaftliche Forschung interessant, sondern insbesondere für Lernende und Lehrende in personenbezogenen Dienstleistungen. Insofern bietet die Einbindung von Learning Analytics in das Serious Game «Take Care» das Potential als Feedback und Anlass zur Reflexion einen Zugewinn hinsichtlich der Wahrnehmung und der Förderung von situativer, hermeneutischer Kompetenz des Einzelfallverstehens zu ermöglichen - ein Ertrag, der letztlich auch von hoher Relevanz für alle weiteren beruflichen Bildungsprozesse in der personenbezogenen Dienstleistung ist. Die Grundfragen von Lernstandsanalysen im Kontext personenbezogener Dienstleistungen mit der Perspektive auf einen belegbaren didaktischen Mehrwert wird das Folgeprojekt LernStandPD im Forschungsverbund differenzierter nachgehen. 


\section{Literatur}

Becker, Bernd. 2013. «Learning Analytics: Insights Into the Natural Learning Behavior of Our Students». Behavioral \& Social Sciences Librarian 32 (1): 63-67. https://doi.org/10.1080/01 639269.2013.751804.

Beutner, Marc, und Rasmus Pechuel. 2015. «App-based Interactive Language Learning - Pedagogy, Implementation and Evaluation with the AGnovel Tool». Proceeding of the E-Learn: World Conference on E-Learning in Corporate, Government, Healthcare, and Higher Education. San Diego: United States Publisher: Association for the Advancement of Computing in Education (AACE). https://www.learntechlib.org/p/152181/.

Breuer, Johannes. 2010. «Spielend lernen? Eine Bestandsaufnahme zum (Digital) Game-Based Learning». Landesanstalt für Medien NRW. https://publikationen.medienanstalt-nrw.de/ index.php?view=product_detail\&product_id=190.

Buck, Günther. 2019. «Teil I Lernen und Erfahrung.»In Lernen und Erfahrung. Epagogik, Phänomenologische Erziehungswissenschaft:3-89. Wiesbaden: Springer Fachmedien. https:// doi.org/10.1007/978-3-658-17098-1_1.

Buckingham Shum, Simon, und Rebecca Ferguson. 2012. «Social Learning Analytics». Educational Technology and Society 15 (3): 3-26. https://www.jstor.org/stable/jeductechsoci.15.3.3.

Chatti, Mohamed Amine, Vlatko Lukarov, Hendrik Thüs, Arham Muslim, Ahmed Muhamed, Fahmy Yousef, Usman Wahid, Christoph Greven, Arnab Chakrabarti, und Ulrik Schroeder. 2014. "Learning Analytics: Challenges and Future Research Directions.» e-learning and education (eleed), 1-16. http://nbn-resolving.de/urn:nbn:de:0009-5-40350.

Darmann, Ingrid. 2004. «POL-transfer durch die Erweiterung von Situationsdeutung». PrinterNet, Nr. 9: 461-67.

Darmann-Finck, Ingrid. 2010. Interaktion im Pflegeunterricht: Begründungslinien der interaktionistischen Pflegedidaktik. IPP-Pflegeforschung, Band 1. Frankfurt am Main: Peter Lang.

DBfK - Deutscher Berufsverband für Pflegeberufe. 2002. «Die Bedeutung professioneller Pflege». https://www.dbfk.de/de/themen/Bedeutung-professioneller-Pflege.php.

Dörner, Ralf, Stefan Göbel, Wolfgang Effelsberg, und Josef Wiemeyer, Hrsg. 2016. Serious Games: Foundations, Concepts and Practice. Switzerland: Springer. https://doi.org/10.1007/978-3319-40612-1.

Dütthorn, Nadin. 2014. Pflegespezifische Kompetenzen im Europäischen Bildungsraum: eine emprische Studie in den Ländern Schottland, Schweiz und Deutschland. Pflegewissenschaft und Pflegebildung, Band 8. Göttingen: V\&R unipress, Universiätsverlag Osnabrück.

Dütthorn, Nadin, und Kathrin Gemballa. 2013. «Theorien und Modelle der Didaktik Ernährung und Hauswirtschaft im Spiegel der Pflegedidaktik.»Bwp@ Spezial, Hochschultage Berufliche Bildung 2013 (Fachtagung 11), hrsg. v. I. Kettschau, S. Stomporowski und K. Gemballa, 1-22. http://www.bwpat.de/ht2013/ft11/duetthorn_gemballa_ft11-ht2013.pdf. 
Erpenbeck, John, Sven Grote, und Werner Sauter. 2017. «Einführung». In Handbuch Kompetenzmessung. Erkennen, verstehen und bewerten von Kompetenzen in der betrieblichen und psychologischen Praxis, herausgegeben von John Erpenbeck, Lutz v. Rosenstiel, Sven Grote, und Werner Sauter, 3. überarbeitete und erweiterte Auflage, IX-XXXVIII. Stuttgart: Schäffer-Poeschel Verlag.

Europäisches Parlament und Rat der europäischen Union. 2016. «VERORDNUNG (EU) 2016/679 DES EUROPÄISCHEN PARLAMENTS UND DES RASTES vom 27. April 2016 zum Schutz natürlicher Personen bei der Verarbeitung personenbezogner Daten zum freien Datenverkehr und zur Aufhebung der Richtlinie 95/46/EG (Datenschutz-Grundverordnung) DSGVO», 27. April 2016. https://eur-lex.europa.eu/legal-content/DE/TXT/PDF/?uri=CELEX:32016R0679.

Ferguson, Rebecca. 2012. «Learning Analytics: Drivers, Developments and Challenges». International Journal of Technology Enhanced Learning 4 (5/6): 304. https://doi.org/10.1504/ IJTEL.2012.051816.

Fromme, Johannes, Ralf Biermann, und Alexander Unger. 2010. «Serious Games» oder «taking games seriouslys?» In Digitale Lernwelten. Konzepte, Beispiele und Perspektiven, herausgegeben von Kai-Uwe Hugger und Markus Walber, 39-57. Wiesbaden: VS Verlag für Sozialwissenschaften. https://doi.org/10.1007/978-3-531-92365-9_3.

Ganguin, Sonja. 2016. «Digitale Spiele. Editorial». merz Wissenschaft medien+erziehung, Zeitschrift für Medienpädagogik 60 (6): 3-10. https://www.merz-zeitschrift.de/alle-ausgaben/ pdf/sonja-ganguin-digitale-spiele/.

Greb, Ulrike. 2010. «Die pflegedidaktische Kategorialanalyse». In Theorien und Modelle der Pflegedidaktik. Eine Einführung, herausgegeben von Roswitha Ertl-Schmuck und Franziska Fichtmüller, 124-63. Weinheim: Juventa-Verlag.

Hoblitz, Anna. 2015. Spielend Lernen im Flow: die motivationale Wirkung von Serious Games im Schulunterricht. Medienbildung und Gesellschaft, Band 33. Wiesbaden: Springer VS. https://doi.org/10.1007/978-3-658-11376-6.

Hülsken-Giesler, Manfred. 2016. «Rekonstruktive Fallarbeit in der Pflege. Ausgangslage und Problemstellung.» In Rekonstruktive Fallarbeit in der Pflege. Methodologische Reflexionen und praktische Relevanz für Pflegewissenschaft, Pflegebildung und die direkte Pflege, herausgegeben von Manfred Hülsken-Giesler, Susanne Kreuzer, und Nadin Dütthorn, 15-32. Pflegewissenschaft und Pflegebildung 13. Göttingen: Vandenhoeck \& Ruprecht.

Hülsken-Giesler, Manfred, Elfriede Brinker-Meyendriesch, Johannes Keogh, Sabine Muths, Margot Sieger, Renate Stemmer, Gudrun Stöcker, und Anja Walter. 2010. «Kerncurriculum Pflegewissenschaft für pflegebezogene Studiengänge - eine Initiative zur Weiterentwicklung der hochschulischen Pflegebildung in Deutschland». Pflege\&Gesellschaft, Zeitschrift für Pflegewissenschaft 3 (15): 216-36.

Hülsken-Giesler, Manfred, und Johannes Koporal. 2013. Fachqualifikationsrahmen Pflege für die hochschulische Bildung: FQR Pflege 6-8. Berlin: Purschke+Hensel.

Hundenborn, Gertrud. 2007. Fallorientierte Didaktik in der Pflege: Grundlagen und Beispiele für Ausbildung und Prüfung. 1. Aufl. München: Elsevier, Urban \& Fischer. 
Ifenthaler, Dirk. 2017. «Are Higher Education Institutions Prepared for Learning Analytics?» TechTrends 61 (4): 366-71. https://doi.org/10.1007/s11528-016-0154-0.

Jung, Eberhard. 2010. Kompetenzerwerb: Grundlagen, Didaktik, Überprüfbarkeit. München: Oldenbourg.

Kerres, Michael. 2018. Mediendidaktik: Konzeption und Entwicklung digitaler Lernangebote. Fifth edition. De Gruyter Studium. Boston, Massachusetts: Walter de Gruyter GmbH.

Kerres, Michael, Mark Bormann, und Marcel Vervenne. 2009. «Didaktische Konzeption von Serious Games: Zur Verknüpfung von Spiel- und Lernangeboten». MedienPädagogik: Zeitschrift für Theorie und Praxis der Medienbildung 2009 (Occasional Papers): 1-16. https:// doi.org/10.21240/mpaed/00/2009.08.25.X.

Kickmeier-Rust, Michael D. 2016. «Support Teachers' Predictions of Learning Success by Structural Competence Modelling.»In Proceedings of the IWTA@EC-TEL 2016, 35-39. Lyon, France. http://ceur-ws.org/Vol-1738/IWTA_2016_paper7.pdf.

Leitner, Philipp, und Martin Ebner. 2017. «Learning Analytics an Hochschulen». In Handbuch Kompetenzentwicklung im Netz. Bausteine einer neuen Lernwelt, herausgegeben von John Erpenbeck und Werner Sauter, 371-86. Stuttgart: Schäffer-Poeschel Verlag.

Liu, Min, Jaejin Lee, Jina Kang, und Sa Liu. 2016. «What We Can Learn from the Data: A Multiple-Case Study Examining Behavior Patterns by Students with Different Characteristics in Using a Serious Game». Technology, Knowledge and Learning 21 (1): 33-57. https://doi. org/10.1007/s10758-015-9263-7.

Pardo, Abelardo, und George Siemens. 2014. «Ethical and Privacy Principles for Learning Analytics: Ethical and Privacy Principles». British Journal of Educational Technology 45 (3): 438-50. https://doi.org/10.1111/bjet.12152.

Schön, Martin, und Martin Ebner. 2013. «Das Gesammelte interpretieren. Educational Data Mining und Learning Analytics». In L3T. Lehrbuch für Lernen und Lehren mit Technologie, herausgegeben von Martin Ebner und Martin Schön. https://l3t.tugraz.at/index.php/LehrbuchEbner10/article/view/119.

Schrader, Claudia. 2010. "Computerbasierte Lernspiele. Stand der Forschung.» In Digitale Spielkultur, herausgegeben von Sonja Ganguin, und Bernward Hoffmann, 179-90. München: Kopaed.

Schulmeister, Rolf. 2007. Grundlagen hypermedialer Lernsysteme: Theorie - Didaktik - Design. 4., überarb. und aktualisierte Aufl. München: Oldenbourg.

Schulmeister, Rolf, Hrsg. 2013. MOOCs - Massive Open Online Courses: offene Bildung oder Geschäftsmodell? Münster: Waxmann.

Staudt, Franz-Peter. 2017. «Workface und Learning Analytics im Arbeitsprozess - Steuerung individueller Arbeits- und Lernprozesse durch die Analyse von Arbeits- und Lernergebnissen». In Handbuch Kompetenzentwicklung im Netz. Bausteine einer neuen Lernwelt, herausgegeben von John Erpenbeck und Werner Sauter, 571-90. Stuttgart: Schäffer-Poeschel Verlag.

Swertz, Christian. 2018. «Bildungstechnologische Echtzeitanalyse». Medienimpulse 56 (1). https://doi.org/10.21243/mi-01-18-02. 
Taraghi, Behnam, Markus Ebner, Martin Ebner, und Martin Schön. 2017. «Learning Analytics an Schulen». In Handbuch Kompetenzentwicklung im Netz. Bausteine einer neuen Lernwelt, herausgegeben von John Erpenbeck und Werner Sauter, 285-301. Stuttgart: Schäffer-Poeschel Verlag.

Unger, Thorsten, Jannis Goossens, und Lisa Becker. 2015. «Digitale Serious Games». In Planspiele und Serious Games in der beruflichen Bildung. Auswahl, Konzepte, Lernarrangements, Erfahrungen - aktueller Katalog für Planspiele und Serious Games, herausgegeben von UIrich Blötz, 5., überarbeitete Auflage, 157-79. Berichte zur beruflichen Bildung. Bonn, Bielefeld: BIBB Bundesinstitut für Berufsbildung, W. Bertelsmann Verlag.

Wang, Hua, Shen Cuihua, und Ute Ritterfeld. 2009. «Enjoyment of Digital Games. Ehat Makes Them «Seriously〉 Fun». In Serious Games. Mechanisms and Effects, herausgegeben von Michael Cody, Peter Vorderer, und Ute Ritterfeld, 25-47. New York: Routledge.

Wechselberger, Ulrich. 2012a. Game-based learning zwischen Spiel und Ernst: das Informations- und Motivationspotenzial von Lernspielen aus handlungstheoretischer Perspektive. München: Kopaed.

Wechselberger, Ulrich. 2012b. «Spielst du noch oder lernst du schon? Der Einfluss des Framings auf Unterhaltung und Inhaltsrezeption bei Game-based Learning». MedienPädagogik: Zeitschrift für Theorie und Praxis der Medienbildung 2012 (Occasional Papers): 1-19. https://doi.org/10.21240/mpaed/00/2012.03.16.X. 